\title{
Simultaneous Poling and Planar Waveguide Fabrication in Glasses
}

\author{
A. L. R. Brennand \\ Instituto de Estudos Avançados, Rd dos Tamoios, Km 5,5, São José dos Campos, São Paulo, CEP 12228-001, Brasil \\ brennand@ieav.cta.br \\ J. S. Wilkinson \\ jsw@ecs.soton.ac.uk \\ School of Electronics and Computer Science, University of Southampton, Southampton SO17 1BJ, United Kingdom
}

\begin{abstract}
Fabrication of buried planar waveguides with $2^{\text {nd }}$ order nonlinear susceptibility in the upper cladding is carried out in soda-lime and BK7 glass substrates in one step by thermal poling. OCIS codes: (190.4390) Nonlinear optics, integrated optics; (230.7390) Waveguides, planar
\end{abstract}

\section{Introduction}

Multicomponent glasses are very versatile materials for integrated optics applications. For instance efficient $\mathrm{Yb} / \mathrm{Er}$ energy transfer and high gain for optical amplification [1], high photosensitivity for grating writing [2], and high $\chi^{(3)}$ for all-optical switching [3], have all been demonstrated in silicate glasses. The latter reveals a potential for realization of high $\chi^{(2)}$ electro-optic waveguides through poling. In principle, all these phenomena may be combined in one substrate in which waveguides can be fabricated to realize a low-cost multifunctional integrated optical technology. In the last two decades a number of techniques, generally known as poling, have been reported to introduce a $2^{\text {nd }}$ order nonlinear susceptibility, $\chi^{(2)}$, into glasses [4-13]. In particular thermal poling of sodium-bearing glasses yields regions with induced 2 nd order nonlinearity, $\chi^{(2)}$, through a "frozen-in" space-charge electric field acting on the inherent third-order nonlinearity, $\chi^{(3)}$. Initially demonstrated in silica glass (Herasil) which has a $\mathrm{Na}^{+}$ ion concentration of tens of ppm [4], thermal poling was also proven to be effective in alkali-rich glasses, where the $\mathrm{Na}^{+}$concentration is up to six orders of magnitude higher than that in Herasil. These included Pyrex [5], soda lime silicate [10], BK7 [11], sodium niobium borophosphate [12] and potassium niobium silicate [13]. The values of $\chi^{(2)}$ of $5.0 \mathrm{pmV}^{-1}$ and $3.8 \mathrm{pmV}^{-1}$ obtained in [12] and [13] respectively yields the potential for practical electrooptic modulation in waveguides made in alkali-rich glasses. In this work the simultaneous formation of optical waveguides with buried cores [14] and 2nd order nonlinear susceptibility, $\chi^{(2)}$, in the upper cladding by thermal poling of alkalirich silicate glasses such as soda lime and BK7 is described.

\section{Experimental}

The fabrication of buried planar waveguides by a constant-current thermal poling procedure in soda-lime (Fisher premium) and BK7 glasses is performed as described in [14]. Samples measuring $25 \mathrm{~mm}$ by $25 \mathrm{~mm}$ by $1 \mathrm{~mm}$ thick were cleaned, and circular 7-mm-diameter aluminum electrodes of thickness $400 \mathrm{~nm}$ were deposited centrally on both faces by vacuum evaporation through a shadow mask. To apply an electric field at elevated temperature we placed each sample in a holder with the cathode pressed onto a silicon wafer, and a high-voltage supply was connected between the anode and the silicon wafer. The assembly was placed in a vacuum chamber with a radiant heater, the chamber was pumped to below $3 \times 10^{-6} \mathrm{mbar}$, and the samples was heated until it reached equilibrium at the desired temperature. The high-voltage supply was then turned on, and a variable voltage was applied so that a constant external current of $20 \mu \mathrm{A}$ was maintained for the process time. Each sample was cooled to room temperature with a constant voltage applied equal to that achieved at the end of the poling process. The external currents fell to zero after approximately 2 min of cooling time. The temperature, current, and applied voltage were continuously recorded from the application of the initial voltage until the samples reached room temperature. The soda-lime and BK7 samples were processed at $200{ }^{\circ} \mathrm{C}$ for $120 \mathrm{~min}$ and at $300{ }^{\circ} \mathrm{C}$ for $180 \mathrm{~min}$, respectively. The voltage applied to maintain a constant current of $20 \mu \mathrm{A}$ rose approximately linearly over the entire duration of the poling process at elevated temperature, as reported in $[10,13,14]$; the applied voltage varied from $90 \mathrm{~V}$ to $2.5 \mathrm{kV}$ for soda-lime glass and from $150 \mathrm{~V}$ to $4.1 \mathrm{kV}$ for BK7 glass. The electrodes were then removed by use of a commercial aluminum etchant and waveguide modes were observed in the region immediately below the removed anodes using the standard prism coupling technique, as reported in [14]. To determine the relative positions of the guiding and of the nonlinear regions the samples were diced and end polished to allow near-field measurements of the modal profiles and 2nd harmonic scanning [15] of the cross-section of the poled glass region underneath the removed anode. Light from a He-Ne laser at a wavelength of $633 \mathrm{~nm}$ was coupled into the waveguides using a monomode optical fiber, and the modal intensity profiles of the waveguide were measured by imaging onto a CCD camera with 
a $63 \times$ objective. The position of the substrate surface was determined by imaging the illuminated end face of the waveguide with the same apparatus. These measurements were calibrated with a micrometric graticule replacing the waveguide edge. Unpolarized mode profiles obtained using this imaging apparatus are shown in Fig. 1 and Fig. 2 for BK7 and soda-lime samples, respectively, with the scales and the absolute positions of the depth axis aligned with an accuracy of $\pm 0.25 \mu \mathrm{m}$, showing that the waveguide mode is buried substantially beneath the substrate surface. A focused Q-switched, mode locked Nd-YAG laser producing a high peak power output with a wavelength of $1.064 \mu \mathrm{m}$ was used for $2^{\text {nd }}$ harmonic generation. The nonlinear region was detected by scanning the cross section of the samples with the focused beam and measuring the reflected $2^{\text {nd }}$ harmonic signal as described in [15]. Fig. 1 and Fig. 2 also show the $2^{\text {nd }}$ harmonic (SH) scanning intensity profiles which show that a $2^{\text {nd }}$ order nonlinear region has been introduced between the surface and the guiding region in the upper cladding of the waveguide.

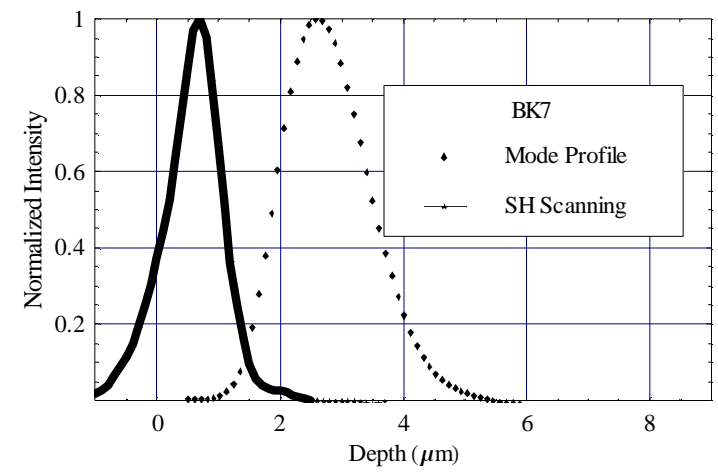

Fig 1. Mode profile and SH profile of a BK7 sample.

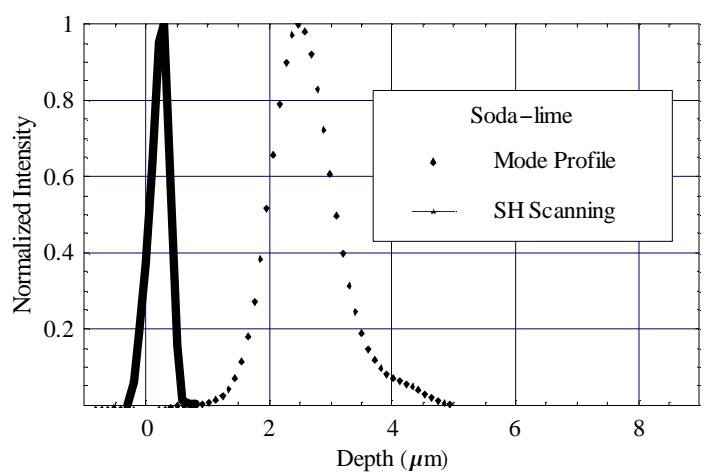

Fig 2. Mode profile and SH profile of a soda-lime sample.

\section{Conclusion}

We have shown that buried planar waveguides with a $2^{\text {nd }}$ order nonlinearity in its upper cladding can be fabricated in one step by a thermal poling procedure. The waveguides are formed by the redistribution of the cations from the surface of the glass to a buried region by differential ionic drift as described in [14]. The resultant electric field due to the depletion of ions from the surface results in a $2^{\text {nd }}$ order nonlinearity in the surface cladding layer. Further experiments are to be carried out for a better characterization of the waveguides and of the nonlinearities obtained and in order to investigate the possibility of electrooptic modulation and $2^{\text {nd }}$ harmonic generation in the waveguide modes.

\section{References}

[1] P. Camy et al, 'Ion-exchanged planar lossless splitter at $1.5 \mu \mathrm{m}$ ', Electron. Lett. Vol. 32, No. 4 (1996).

[2] D.F. Geraghty, D. Provenzano, M.M. Morrell, J. Ingenhoff, B. Drapp, S. Honkanen, A. Yariv, N. Peyghambarian, 'Polarisation-independent Bragg gratings in ion-exchanged glass channel waveguides', Electron. Lett. Vol 36, No. 4 (2000).

[3] J.S. Aitchison et al, 'The nonlinear optical properties of glass', Metals Materials and Processes, Vol. 8, No. 4, 277-290 (1996).

[4] R.A. Myers, N. Mukherjee, and S.R.J. Brueck, 'Large 2nd -order nonlinearity in poled fused silica', Opt. Lett., Vol. 16, 1732 (1991).

[5] A. Okada et al, 'Phase-matched 2nd -harmonic generation in novel corona poled glass waveguides', Appl. Phys. Lett. Vol. 60, No. 23, 1992).

[6] A.C. Liu et al, 'Electro-optic phase modulation in a silica channel waveguide', Opt. Lett.. Vol. 19, No. 7, (April 1994).

[7] L. J. Henry et al, 'Optical nonlinearity in fused silica by proton implantation', J. Opt. Soc. Am. B. Vol.13, No 5, 827 (1996)

[8] M. Abe, T. Kitagawa, K. Hattori, A. Himeno and Y. Ohmori, 'Electro-optic switch constructed with a poled silica based waveguide on a Si substrate', Elect. Lett., Vol. 32, No. 10, (May 1996).

[9] T. Fujiwara, M. Takahashi, and A.J. Ikushima, '2nd -harmonic generation in UV-poled Germanosilicate glass', ECOC 97, 22-25 September 1997, Conference Publication No 448.

[10] F.C. Garcia et al, 'Inducing a large 2nd -order optical nonlinearity in soft glasses by poling', Appl. Phys. Lett., Vol. 72 , No. 25 (22 Jun 1998).

[11] M. Qiu, T. Mizunami, R. Vilaseca, F. Pi and G. Orriols, 'Bulk and near-surface 2nd -order nonlinearities generated in a BK7 soft glass by thermal poling', J. Opt. Soc. Am. B, Vol. 19, No. 1 (Jan 2002).

[12] M. Dussauze, E. Fargin, M. Lahaye, V. Rodriguez, F. Adamietz, Large 2nd -harmonic generation of thermally poled sodium borophosphate glasses, Opt. Express 13 (May 2005) 4065.

[13]P. Pernice et al, Electric field induced structural modification and 2nd order optical nonlinearity in potassium niobium silicate glass, J. NonCryst. Solids 355 (2009) 2578.

[14] A. L. R. Brennand and J. S. Wilkinson, 'Planar waveguides in multicomponent glasses fabricated by field-driven differential drift of cations', Opt. Lett. Vol.27, No. 11 (June 2002).

[15] J. Arentoft et al, '2nd -harmonic imaging of poled silica waveguides', Appl. Phys. Lett. Vol. 76, No. 1 (Jan 2000).

[16] A. L. R. Brennand, J. S. Wilkinson and P. G. Kazansky, 'Evaluation of alkali-rich glasses for poling', CLEO 19-24 May 2002, Technical Digest, 236. 